MODELING, IDENTIFICATION AND CONTROL, 1994, VOL. 15, NO. 3, 135-139

doi:10.4173/mic.1994.3.2

\title{
"Every good regulator of a system must be a model of that system"
}

\author{
PIETER EYKHOFF $\dagger$
}

Keywords: Modeling, identification, and control

A model for the process under control-do or don't we really need it? Some elementary philosophical considerations confirming such a need, are well supported by examples of various 'optimal' control schemes. How does this affirmation influence the requirements to identification and the implementation of control using such a model?

\section{Introduction}

Dear reader, you are perfectly right: the title of the contribution is not original; ... hence the use of quotation-marks. This title is identical to that of a paper by Conant and Ashby (1970). Don't you think it is a challenging statement? So do I. But, judging from the citation index, that paper received (far too) little attention; the control engineering community in particular responded sluggishly to this challenge.

While offering my apologies for the 'borrowing' just confessed, I also would like to express as my opinion that this title might be interpreted as strong support for the Editorial Board and the Editor of this journal. Fifteen years ago they chose the name 'Modeling, Identification and Control. This choice can be seen as a statement of a professional conviction; it emphasizes their views on the crucial importance of the combination of these three aspects for control engineering and for its development. In this note we will study to what, degree this conviction was right.

\section{The essence of feedback}

"Every good regulator of a system must be a model of that system"? Probably no small number of control engineers, devoted to the successful PID-controllers as the real workhorses of automatic control in practice, might meet this statement with a howl of anguish.

What 'fool' would be prepared to defend the given proposition? Who recognizes in the (numerical values of the) P-, I- D-terms of a controller a fair, or even a roughly approximated model of the complex chemical/physical/electrical/...p process that is being controlled by that controller?

Let us walk another trail; let us look at the essence of control principles in an old, simple and quite fundamental way (Smith, 1958). Figure 1, by means of block diagram substitutions through the sequence a, b, c, d, gives a development of the control scheme for response improvement by feed back. In this scheme $H_{\mathrm{p}}$ viz. $H_{\mathrm{M}}$ represent the process, viz. the model dynamics. The $\infty$ sign indicates an 'operational amplifier' with infinite gain. The essential aspect of this reasoning is the role that is played by the model

Received 25 January 1994.

$\dagger$ Department of Electrical Engineering, Eindhoven University of Technology, NL-5600 MB Eindhoven, The Netherlands. 


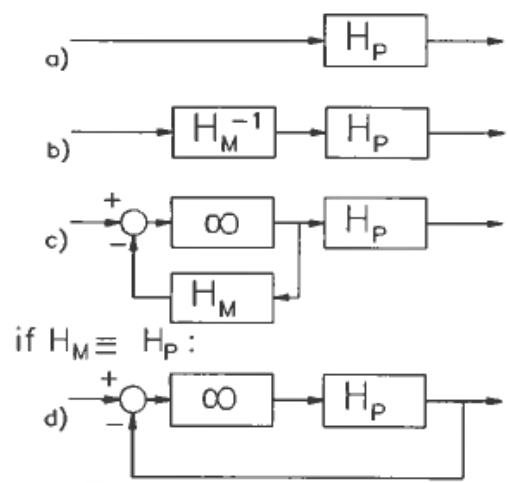

Figure 1. Development of a control scheme for response improvement.

$H_{\mathrm{M}}$. It is clear that for the case $H_{\mathrm{M}}=H_{\mathrm{p}}$ the process acts as its own model, i.e. no separate model is needed!

Similar fundamental lines of thought hold for other control schemes like:

- disturbance compensation by feed forward,

- disturbance compensation by feed back, and

- predictor control of a process with time delay.

For the purpose of this discourse there is no need to enter into a discussion on the limitations of this type of reasoning in practice. It is evident that in real life the ideal situation represented by such schemes can only be approximated because of the restrictions on feasibility and quality of the $H_{\mathrm{M}}^{-1}$ approximation; the restrictions on the components, e.g. their non-linear behaviour; the restrictions on amplitudes and speed of changes of the input due to the need for a 'smooth' operation of the process, etc. However, from such examples the potential role of models is clear, in spite of these practical limitations.

\section{Various 'optimal' control schemes}

As a next step in exploring the proposition given by the title of this paper, it would be of interest to analyse a substantial part of the numerous control schemes that have been proposed in literature. Do they or don't they, explicitly or implicitly, use a 'model' of the process under control? And, if they do, what model-structure is required?

The size allowed for this contribution does not permit an extensive analysis. For the limited objective we have in mind, the following approach might be acceptable. Figures 2 and 3 give examples of very simple processes, as well as the associated controllers that result from particular 'optimal' control criteria:

\begin{tabular}{lll}
\hline Fig & Control criteria & Process \\
\hline 2a & minimum variance & pure time delay \\
2b & - & $\begin{array}{l}\text { 2nd order dynamics + delay } \\
\text { do., + zero in right H.P. }\end{array}$ \\
2c & - & 1st order dynamics \\
2d & dead beat & 1st order dynamics \\
3a & $\begin{array}{l}\text { min. integral with } \\
\text { input constraints }\end{array}$ & 1st order dynamics \\
3b & time-optimal & 2nd order dynamics \\
3c & - & linear dynamics \\
3d & linear quadratic & \\
\hline
\end{tabular}




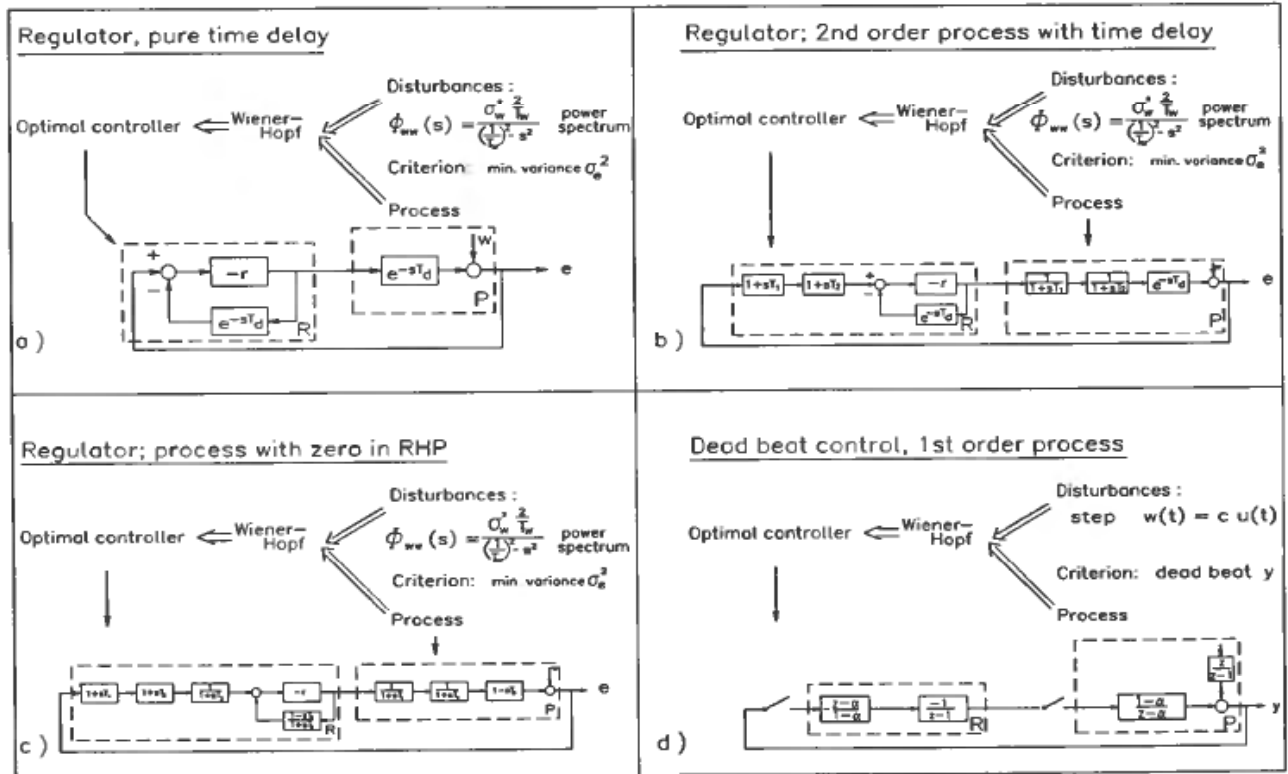

Figure 2. Examples of controllers for various 'optimal' control criteria-I.

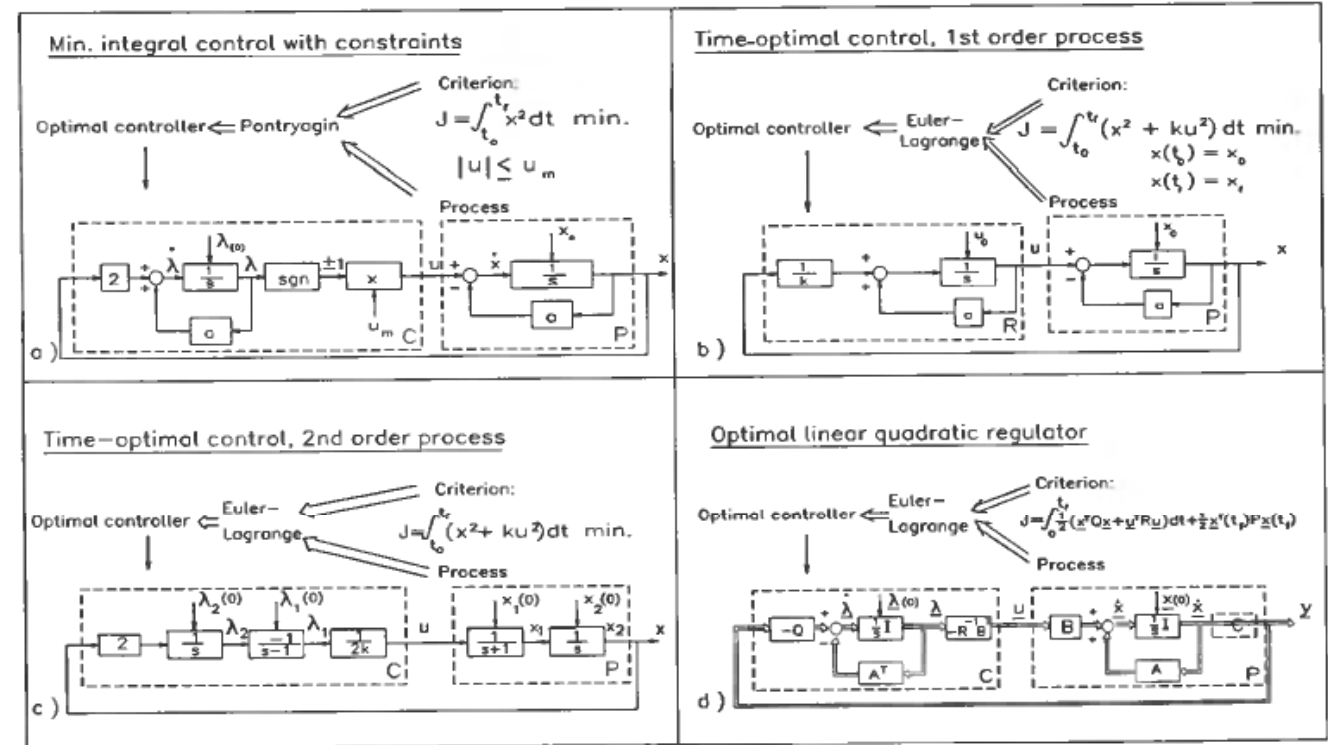

Figure 3. Examples of controllers for various 'optimal' control criteria-II. 
These simple diagrams, which take into account invertibility restrictions on dynamics, clearly indicate the similarity between the process dynamics and the corresponding controller. Apparently the proposition posed by the title holds very well indeed...

\section{Relation between control theory and engineering}

In the previous examples the process was assumed to be known completely and accurately, i.e. it was presumed that no information on parts be missing and that no errors do occur in the parameter values. These assumptions constitute the difference between practice and theory, between reality and model. This is expressed in Fig. 4, which suggests the control theory can be characterized by application of control principles (algorithms) to well-known, well-specified, well-behaving models, and control engineering by the application of such algorithms to reality, i.e. to partly and incompletely known processes. As the projection reality $\rightarrow$ model is supported by identification, this technique is bound to play a role of increasing importance; more and more it will contribute to bridging the gap between 'theory' and 'engineering'. This is schematically indicated in Fig. 4 by curve $B$.

Since the early stages in the development of identification (Eykhoff, 1974) much theory, many tools (computer software) and a wide variety of applications have been developed. The techniques have successfully been used in real industrial situations. Even under such challenging conditions the internal model control (IMC) scheme has demonstrated to be an effective expedient for improving disturbance reduction, for realizing decoupling between various input-output relations, as well as for speeding up system dynamics, i.e. reducing change-over time for set point changes (Zhu and Backx, 1993).

Of course the last observation does not imply that all (classes of related) problems are solved and that we have all the answers; that is not the case, even not in principle. More insight is needed in at least the following aspects:

- the best type of model and its (unavoidable) model uncertainty for particular control schemes, e.g. for robust $H \propto$ control;

- the appropriate ways to introduce into the model the kind of a priori knowledge that is available on the process;

- the relation between complexity of the model and the quality of control;

- the limitations on the speed of identification under specific (practical) circumstances, etc.

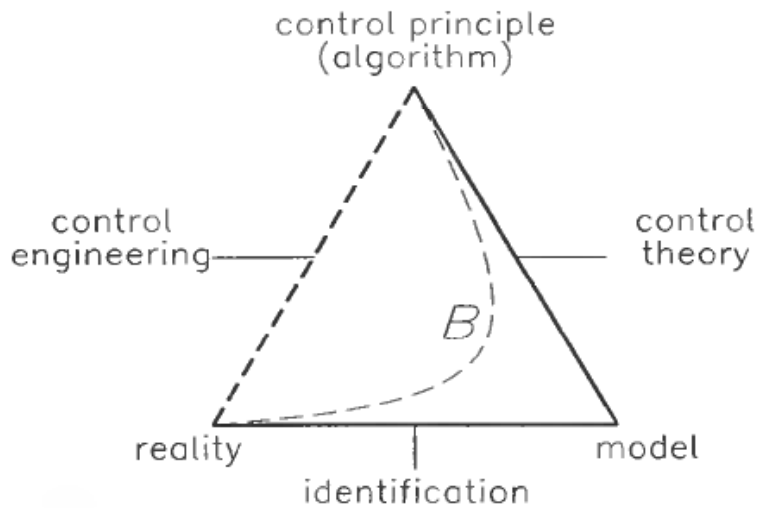

Figure 4. Modeling, identification and control. 
For that reason we do not need a crystal ball in order to predict a continuing research along such paths. This holds the more as both theory and practice have a joint interest in such developments.

\section{Conclusions}

From the foregoing considerations the conclusion is clear: a compact and accurate process model is the key for the application (to industrial processes) of modern control theory. Hence in essence the statement "Every good regulator of a system must be a model of that system" reflects a correct and fruitful philosophy in control engineering;

Fifteen years ago the Editorial Board and the Editor of this journal were perfectly right in choosing the name 'Modeling, Identification and Control (MIC)' for their journal, witnessing the crucial role these three elements and their interaction play.

The result attained so far in this field, the challenges available these days, and the tempting promises for the future provide ample reasons to predict a healthy development and a useful role for $M I C$ over a period of, at least, another fifteen years. Congratulations, best wishes to $M I C$, its Editor and its Editorial Board...

\section{REFERENCES}

CONANT, R. C., and AsHBY, W. R. (1970). Every good regulator of a system must be a model of that system. Int. J. Systems Sci., 1, 89-97.

Sмiтн, O. J. M. (1958). Feedback Control Systems (McGraw-Hill, New York).

EykнOF, P. (1974). System Identification; Parameter and State Estimation (Wiley, Chichester).

ZHU, Y., and BACKX, T. (1993). Identification of Multivariable Industrial Processes (for Simulation, Diagnosis and Control) (Springer-Verlag, London). 